Интернет-журнал «Науковедение» ISSN 2223-5167 http://naukovedenie.ru/

Том 7, №1 (2015) http://naukovedenie.ru/index.php?p=vol7-1

URL статьи: http://naukovedenie.ru/PDF/93TVN115.pdf

DOI: 10.15862/93TVN115 (https://doi.org/10.15862/93TVN115)

УДК 621.314.572

Коптяев Евгений Николаевич

ОАО «Северное Производственное Объединение «Арктика»

Российская Федерация, Северодвинск ${ }^{1}$

Инженер по наладке и испытаниям 1 категории

Старший преподаватель

E-mail: evgenijkoptjaev@gmail.com

РИНЦ: http://elibrary.ru/author_profile.asp?id=792304

Балашевич Вячеслав Михайлович

НИИ Энергетики ЮРГТУ

Российская Федерация, г. Новочеркасск ${ }^{2}$

Ведущий инженер

E-mail: bmashka@gmail.com

Атрашкевич Павел Васильевич

ОАО «Северное Производственное Объединение «Арктика»

Российская Федерация, Северодвинск

Инженер по наладке и испытаниям 1 категории

E-mail: bimfin@mail.ru

Душкин Юрий Владимирович

ОАО «Северное Производственное Объединение «Арктика»

Российская Федерация, Северодвинск

Заместитель главного инженера

E-mail: yuri.dushkin@yandex.ru

Кузнецов Иван Васильевич

ОАО «Производственное Объединение «Севмаш»

Российская Федерация, Северодвинск ${ }^{3}$

Мастер

Старший преподаватель

Аспирант

E-mail: fenix-north@ya.ru

1 164500, Северодвинск, Архангельской области, Архангельское шоссе, 34

2 346428, Ростовская область г. Новочеркасск, ул. Просвещения, 132

${ }^{3}$ 164500, Северодвинск, Архангельской области, Архангельское шоссе, 58 


\section{Трансформатор с вращающимся магнитным полем как структура с дополнительной степенью свободы}

Аннотация. Рассмотрен трансформатор с вращающимся магнитным полем как электротехническая система с дополнительной степенью свободы, что определяет его уникальные возможности по преобразованию выходного напряжения. Проведен анализ развития конструкций трансформаторов, в том числе конструкций трансформаторов с пульсирующим магнитным полем с пространственной магнитной системой, и появления трансформаторов и реакторов с вращающимся магнитным полем, а также ферромагнитных умножителей частоты. Сделано заключение, что наличие дополнительной степени свободы и пространственное перемещение суммарного магнитного поля трехфазной первичной обмотки позволяет преобразовывать частоту ЭДС вторичной обмотки. Описан случай умножения частоты выходного напряжения в трансформаторе с вращающимся магнитным полем, приведена осциллограмма напряжения на выходе умножителя частоты на базе трансформатора с вращающимся магнитным полем.

Ключевые слова: трансформатор с вращающимся магнитным полем; многомерная система; степень свободы; зубцовые гармоники; преобразование частоты; трансформатор частоты; магнитопровод; ферромагнитный умножитель частоты.

Ссылка для цитирования этой статьи:

Коптяев Е.Н., Балашевич В.М., Атрашкевич П.В., Душкин Ю.В., Кузнецов И.В. Трансформатор с вращающимся магнитным полем как структура с дополнительной степенью свободы // Интернет-журнал «НАУКОВЕДЕНИЕ» Том 7, №1 (2015) http://naukovedenie.ru/PDF/93T VN115.pdf (доступ свободный). Загл. с экрана. Яз. рус., англ. DOI: 10.15862/93T VN115 
Согласно ГОСТ16110-82 трансформатором называется статическое электромагнитное устройство, имеющее несколько (две или более) индуктивно связанных обмоток и предназначенное для преобразования посредством электромагнитной индукции одной или нескольких систем переменного тока на входе в одну или несколько систем переменного тока на выходе. Известны трансформаторы напряжения и тока, в основе которых лежит закон электромагнитной индукции. При этом в широком смысле, трансформаторы, как и электромашинные преобразователями, можно считать разновидностью индуктивной электрической машины переменного тока [8]. Теория электромашинных преобразователей хорошо проработана и в обобщенном виде представлена, например в $[8,11,14]$. Принцип действия электромеханических преобразователей можно описать универсальной энергетической функцией [14]:

$$
\ni(t) \delta t=\sum_{1}^{N} \delta\left\{\oint m_{i} \vec{\vartheta}_{\imath} d \overrightarrow{l_{l}}+\iint d \psi_{i} d q_{i}\right\}
$$

где $\mathrm{N}$ - число элементов (материальных контуров тока) системы;

$m_{i}-$ масса; $\overrightarrow{\vartheta_{l}}-$ скорость перемещения; $\psi_{i}-$ магнитное потокосцепление электрического тока і -го элемента системы.

Отсюда следует основное отличие электромашинных преобразователей от трансформаторов - в наличии периодического механического перемещения вращающихся относительно друг друга обмоток, а значит, и взаимных индуктивностей, что позволяет создавать преобразователи электрической энергии одних параметров в другие, при этом возможно преобразование напряжения, тока и частоты одновременно. Трансформаторы лишены первого из слагаемых, и произведение $m_{i} \overrightarrow{\vartheta_{l}} d \vec{l}_{l}$ для них обращается в нуль, и функция интенсивности энергетического взаимодействия определяется только потокосцеплением электрического тока і-го элемента системы.

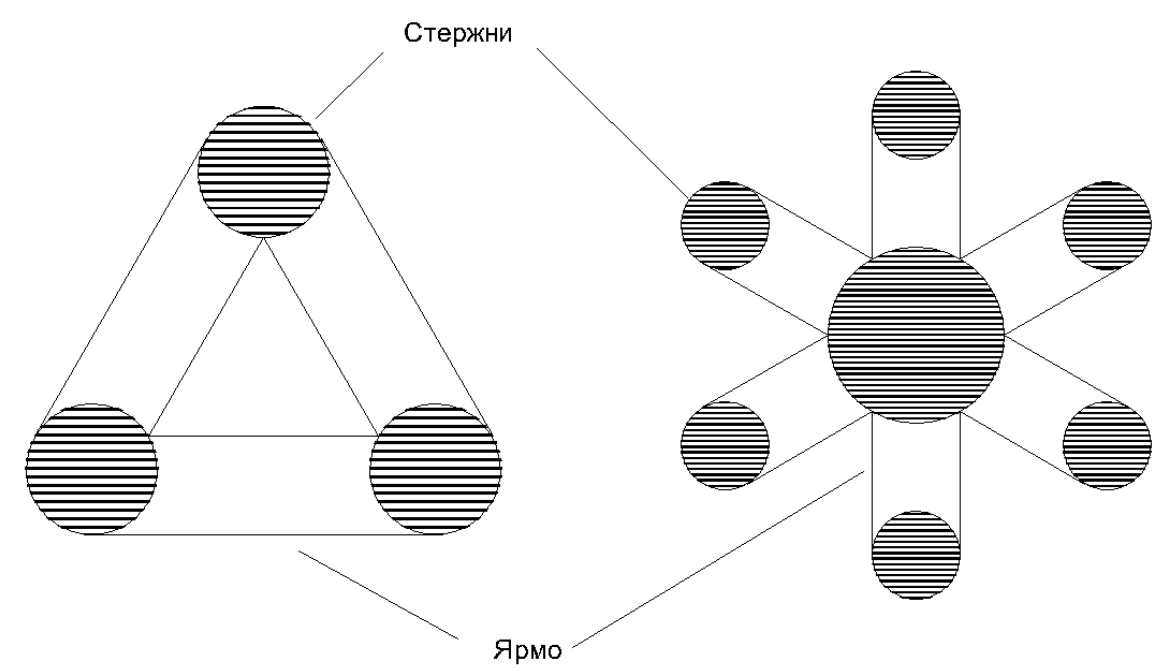

Рисунок 1. Разновидности пространственных магнитных систем трансформаторов с пульсирующим магнитным полем 
Применяемые в электроэнергетике силовые трансформаторы представляют собой чаще всего плоские магнитные системы стержневого типа со ступенчатой формой сечения стержней. В настоящее время выпускаются трансформаторы самого различного назначения и широким диапазоном мощностей вплоть до 1 млн. кВ·А [7]. К достоинствам классических трансформаторов с пульсирующим магнитным полем можно отнести простоту конструкции, удобство изготовления и технологии сборки. При этом магнитная система таких устройств является принципиально несимметричной, что является причиной возникновения высших гармоник в магнитном потоке и ухудшению качества выходного напряжения и потребляемого из сети тока [10]. Как способ улучшения качества гармонического состава магнитного потока трансформаторов, появились пространственные магнитные системы, в которых все стержни имеют равные размеры и форму, а также симметричное расположение [7]. Основные возможные варианты построения симметричной пространственной магнитной системы приведены на рис.1.

В целом, силовые трансформаторы с пульсирующим магнитным полем (ТПМП) можно рассматривать как систему с двумя степенями свободы (с точки зрения распределения магнитного потока): рабочий магнитный поток протекает в одной плоскости, не меняя своего положения; переменная составляющая переменного тока первичной обмотки заключается в изменении (пульсировании) значения индукции в стержнях магнитопровода.

Дальнейшим развитием конструкции трансформаторов стало появление трансформаторов и реакторов с вращающимся магнитным полем, обладающих симметричной магнитной системой и синусоидальным током, потребляемым из сети $[1,2,3,12]$. Их конструкция в основном аналогична электрическим машинам переменного тока с заторможенным ротором и пространственно-распределенной трехфазной первичной обмоткой, обладающей круговой симметрией. В трансформаторах с вращающимся магнитным полем (ТВМП) возможно использование наработок и методов расчета, разработанных в теории электромашинных преобразователей в части формирования первичной трехфазной (многофазной) сетевой обмотки. В качестве вторичной обмотки используются два принципиально разных типа обмоток: распределенная трехфазная (многофазная) с включением фаз звездой или треугольником, а также замкнутая круговая обмотка (КО). Оба варианта имеют свои достоинства и недостатки - повышенный расход меди при увеличении числа фаз и наличие уравнительного тока при различного рода несимметриях для КО соответственно. Трансформаторы с КО нашли применение в полупроводниковых статических выпрямителях [4, 5] и инверторах [6], обладающих повышенным качеством выходного напряжения и электромагнитной совместимостью с питающей сетью. Во всех этих решениях трансформатор с вращающимся магнитным полем используется в качестве умножителя числа фаз системы для улучшения качества выходного напряжения управляемых выпрямителей или инверторов. Также применяется ТВМП с несколькими вторичными трехфазными обмотками[3], сдвинутым конструктивно относительно друг друга - что обеспечивает фазовый сдвиг их ЭДС (такое решение было использовано ОАО «Электросила» для снижения массогабаритных показателей мощных трансформаторов) без использования соединения обмоток в треугольник, требующий в $\sqrt{3}$ раз большее число витков.

К сожалению, до сих пор трансформаторы с вращающимся магнитным полем можно признать до сих пор недооцененными отечественной промышленностью. Хотя за два последних десятилетия сформировался ряд научных коллективов, занимавшихся конструкцией ТВМП. Например, работы $[1,2,3]$, а также $[4,5,6,12]$ и также ряд других исследований $[9,13]$. Примеров их внедрения в промышленности не так много. Их усилия сосредоточились на трансформаторе с вращающимся полем как системе с симметричными магнитными и электрическими параметрами [1], и конструкции ТВМП с распределенной многофазной круговой обмоткой[4,5,6]. Тем не менее, до сих пор ТВМП - не более чем умножитель числа 
фаз выходного напряжения. Симметрия КО позволяет наращивать число фаз выходного напряжения без ухудшения массы и габаритов, что может быть удобно в многофазных статических выпрямителях, но при этом она неприменима для питания многофазной нагрузки переменного тока.

Подобную ситуацию, отчасти, можно связать с некоторым недопониманием физической сущности трансформаторов, а именно - наличием дополнительной степени свободы. Что это значит? Обратимся к эволюции математической модели обобщенной электрической машины [11], частным случаем которой является индуктивный преобразователь переменного тока (трансформатор). Простейшим состоянием электромагнитного поля является пульсирующее поле неподвижной одиночной катушки; следующая ступень - круговое поле однофазной электрической машины, при этом, если её ротор неподвижен - конструкция соответствует катушке с броневой конструкцией магнитопровода; далее следует вращающееся магнитное поле - образованное m-фазной системой ЭДС. В основе всех этих вариаций лежит разное число степеней свободы. Для обобщенного описания используются системы уравнений, так что в общем случае, число уравнений пропорционально числу степеней свободы рассматриваемой системы. Наибольшее число уравнений у многофазной электрической машины с вращающимся ротором [11].

Трансформатор с вращающимся магнитным полем является многофазной электрической машиной с заторможенным ротором. Отсутствие механического перемещения приводит к снижению степеней свободы, и как следствие - к упрощению математической модели и уменьшению числа уравнений, описывающих систему. Тем не менее, их число больше, чем в трансформаторах с пульсирующим магнитным полем, где нет механического перемещения а магнитные величины описываются законами изменения во времени.

Обратимся к физическому смыслу вращающегося магнитного поля. Как известно, первичная трехфазная обмотка ТВМП создает вращающееся магнитное поле, так что закон изменения трехфазных токов в фазах обмотки определяет пространственное распределение во времени намагничивающих сил, при этом электромагнитное поле не пульсирует - a перемещается в пространстве, численное же его значение постоянно. Сохраняется аналогия с электромеханическими преобразователями электроэнергии, но из системы уравнений исчезает переменная $\frac{d \psi}{d t}$, становясь равной нулю, а угол поворота обмоток $\alpha$ всегда постоянен. Остается пространственное перемещение суммарного магнитного поля трехфазной первичной обмотки, что позволяет преобразовывать частоту ЭДС вторичной обмотки. При этом отсутствие изменения потокосцеплений контуров не позволяет плавно регулировать выходную частоту, как следствие снижения степени свободы системы. Подобные преобразователи частоты можно назвать трансформаторами частоты - по основному изменяемому параметру. В отличие от трансформаторов для преобразования частоты $[8,13]$ (иначе - ферромагнитные умножители частоты), где в пульсирующем магнитном потоке выделяются высшие (например, третья) гармоники МДС с крайне низкой эффективностью, трансформатор частоты отличает лучшие масса, габариты и энергетические параметры [14]. 


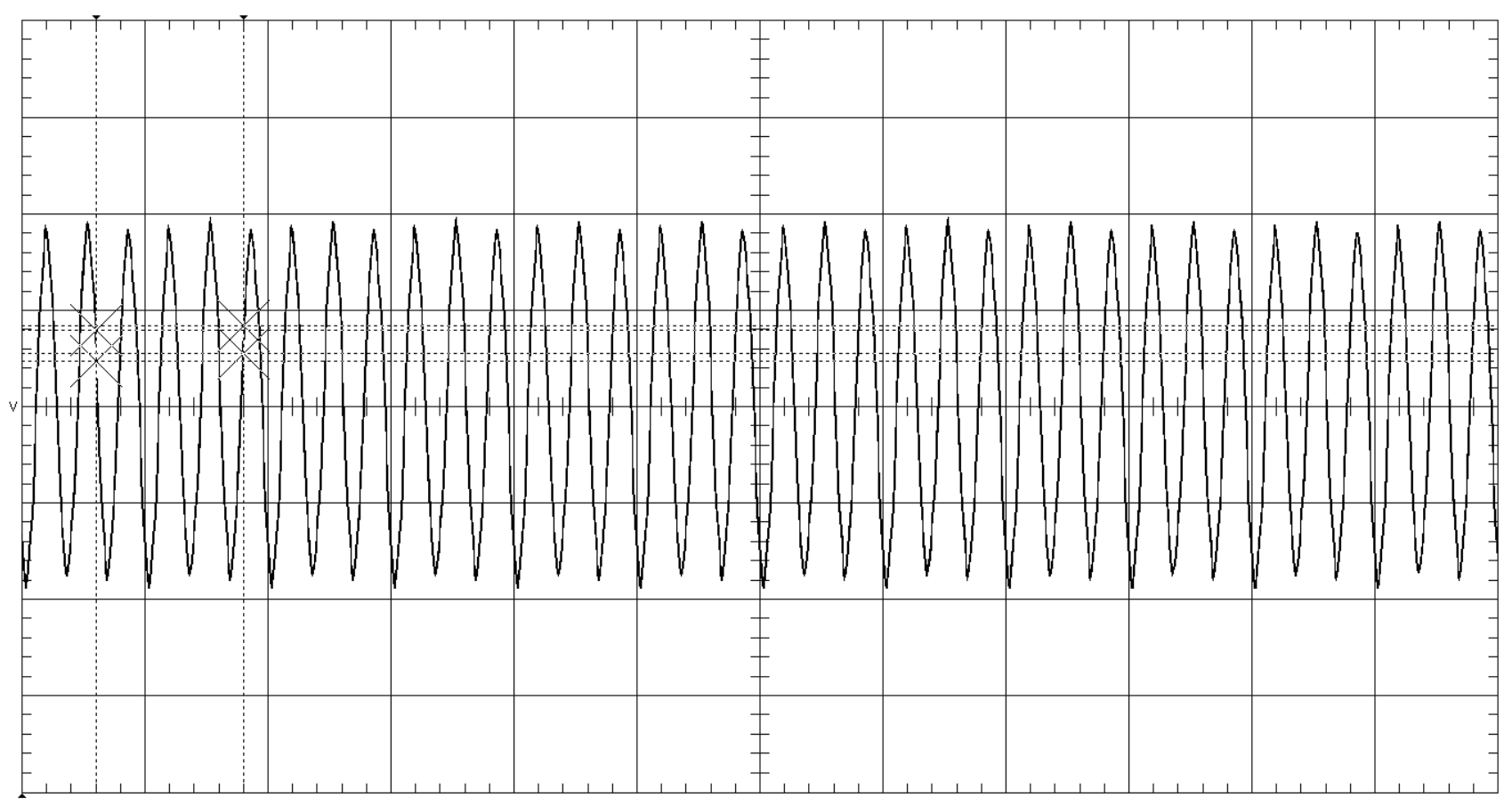

Рисунок 2. Осииллограмма выходного напряжения трансформатора частоты с 3-кратным умножением

Проведенные в ОАО «СПО «Арктика», испытания опытных образцов трансформаторов частоты с 3, 6 и 8 -кратным умножением подтвердили их промышленную применимость.

\section{Выводы:}

1 Трансформаторы с вращающимся магнитным полем обладают не только распределенной магнитной цепью, но и являются системой с дополнительной степенью свободы;

2 Применение трансформаторов частоты способно частично заменить статические полупроводниковые преобразователи, при отсутствии требования плавного регулирования частоты напряжения на выходе. 


\section{ЛИТЕРАТУРА}

1. Атрощенко В.А., Гайтов Б.Х., Сингаевский Н.А., Луков Ф.И. Гармонический анализ кривой МДС трансформатора с вращающимся магнитным полем Электромеханика, 1997, №1, с. 9.

2. Гайтов Б.Х., Прасько Д.Г., Гайтова Т.Б Разработка многофазных трансформаторов - Электротехника, 2000, №8, с. 42.

3. Гайтов Б.Х., Гайтова Т.Б., Кашин Я.М. Перспективные конструкции аксиальных многофазных трансформаторов и регуляторов с вращающимся магнитным полем - Электромеханика, 2005, №3, с. 44.

4. Черевко А.И., Семенов Д.Н., Казакевич А.И. Схемотехнические модели выпрямителей с трансформатором вращающегося магнитного поля в MICROСАР 7 - Электротехника, 2005, №4, с. 8.

5. Черевко А.И., Музыка М.М., Лимонникова Е.В. Влияние силовых схем коммутаторов на качество выходного напряжения управляемых выпрямителей, построенных на базе трансформаторов с вращающимися магнитными полями. Электротехника, 2008, №10, с. 22.

6. Черевко А.И., Музыка М.М. Исследование качества выходного напряжения, тока и энергетических соотношений автономных инверторов с трансформаторами вращающегося магнитного поля. - Электротехника, 2009, №2, с. 56.

7. Тихомиров П.М. Расчет трансформаторов. - Учебное пособие для вузов. М.: Альянс, 2013, 528 с.

8. Иванов - Смоленский А.В. Электрические машины: учебник для вузов. В двух томах. Том 1. - М.: Издательство МЭИ, 2006, 652 с.

9. З Забудский Е.И. Совмещенные регулируемые реакторы. - М.: ФГОУ ВПО МГАУ, Энергоатомиздат, 2003, 436 с.

10. Забудский Е.И. Электрические машины. Ч. 1. Трансформаторы. Учебное пособие для вузов. Москва: МГАУ, 2002, 167 с.

11. Копылов И.П. Обобщенная электрическая машина и обобщенный электромеханический преобразователь. - Электротехника, 2008, №2, с. 50.

12. Черевко А.И., Дмитриев Б.Ф., Рябенький В.М., Музыка М.М., Солуянов П.В. Судовые полупроводниковые преобразователи. - СПб: СПбГМТУ, 2011, 526 с.

13. Загрядцкий В.И., Кобыляцкий Н.И., Недзельский А.П. Ферромагнитные умножители частоты с вращающимся магнитным полем. Кишинев: «Картя Молдовеняскэ», 1973, 71 с.

14. Копылов И.П., Гандилян С.В., Гандилян В.В. Некоторые вопросы обобщенного физико-математического моделирования электромеханических преобразователей энергии - Электротехника, 1998, №9, с. 25.

Рецензент: Каплин, зам. главного конструктора по специальной тематике, кандидат технических наук, НИИ Энергетики ЮрГТУ. 
Koptjaev Evgenij Nikolaevich

JSC "Northern Industrial Association" Arctic"

Russian Federation, Severodvinsk

E-mail: evgkop79@mail.ru

\author{
Balashevich Vyacheslav Mikhailovich \\ Energy Research Institute YURGPU \\ Russian Federation, Novocherkassk \\ E-mail: bmashka@gmail.com
}

Atrashkevich Pavel Vasilevich

JSC "Northern Industrial Association" Arctic"

Russian Federation, Severodvinsk

E-mail: bimfin@mail.ru

Dushkin Jurij Vladimirovich

JSC "Northern Industrial Association" Arctic"

Russian Federation, Severodvinsk

E-mail: yuri.dushkin@yandex.ru

Kuznetsov Ivan Vasilyevich JSC "Industrial Association"Sevmash"

Russian Federation, Severodvinsk

E-mail: fenix-north@ya.ru

\title{
A transformer with a rotating magnetic field as a structure with an additional degree of freedom
}

\begin{abstract}
Considered a transformer with a rotating magnetic field as electrical system with an additional degree of freedom, which determines its unique opportunity to transform the output voltage. An analysis of structures of transformers, including transformer design with pulsed magnetic field with a spatial magnetic system and the appearance of transformers and reactors with a rotating magnetic field and the ferromagnetic frequency multipliers. It is concluded that the presence of additional degrees of freedom and the spatial displacement of the total magnetic field of three-phase primary winding can convert EMF frequency of the secondary winding. A case of multiplying the frequency of the output voltage of the transformer with a rotating magnetic field, shows an oscillogram of the voltage at the output of the frequency multiplier based on a transformer with a rotating magnetic field.

Keywords: transformer with a rotating magnetic field multidimensional system; the degree of freedom the claw harmonic frequency conversion; frequency transformer; magnetic; ferromagnetic frequency multiplier.
\end{abstract}




\section{REFERENCES}

1. Atroshchenko V.A., Gaytov B.Kh., Singaevskiy N.A., Lukov F.I. Garmonicheskiy analiz krivoy MDS transformatora $\mathrm{s}$ vrashchayushchimsya magnitnym polem Elektromekhanika, 1997, №1, s. 9.

2. Gaytov B.Kh., Pras'ko D.G., Gaytova T.B Razrabotka mnogofaznykh transformatorov - Elektrotekhnika, 2000, №8, s. 42.

3. Gaytov B.Kh., Gaytova T.B., Kashin Ya.M. Perspektivnye konstruktsii aksial'nykh mnogofaznykh transformatorov i regulyatorov s vrashchayushchimsya magnitnym polem - Elektromekhanika, 2005, №3, s. 44.

4. Cherevko A.I., Semenov D.N., Kazakevich A.I. Skhemotekhnicheskie modeli vypryamiteley s transformatorom vrashchayushchegosya magnitnogo polya v MICROCAP 7 - Elektrotekhnika, 2005, №4, s. 8.

5. Cherevko A.I., Muzyka M.M., Limonnikova E.V. Vliyanie silovykh skhem kommutatorov na kachestvo vykhodnogo napryazheniya upravlyaemykh vypryamiteley, postroennykh na baze transformatorov $\mathrm{s}$ vrashchayushchimisya magnitnymi polyami. - Elektrotekhnika, 2008, №10, s. 22.

6. Cherevko A.I., Muzyka M.M. Issledovanie kachestva vykhodnogo napryazheniya, toka i energeticheskikh sootnosheniy avtonomnykh invertorov $s$ transformatorami vrashchayushchegosya magnitnogo polya. - Elektrotekhnika, 2009, №2, s. 56.

7. Tikhomirov P.M. Raschet transformatorov. - Uchebnoe posobie dlya vuzov. M.: Al'yans, 2013, $528 \mathrm{~s}$.

8. Ivanov - Smolenskiy A.V. Elektricheskie mashiny: uchebnik dlya vuzov. V dvukh tomakh. Tom 1. - M.: Izdatel'stvo MEI, 2006, $652 \mathrm{~s}$.

9. Zabudskiy E.I. Sovmeshchennye reguliruemye reaktory. - M.: FGOU VPO MGAU, Energoatomizdat, 2003, $436 \mathrm{~s}$.

10. Zabudskiy E.I. Elektricheskie mashiny. Ch. 1. Transformatory. Uchebnoe posobie dlya vuzov. Moskva: MGAU, 2002, 167 s.

11. Kopylov I.P. Obobshchennaya elektricheskaya mashina i obobshchennyy elektromekhanicheskiy preobrazovatel'. - Elektrotekhnika, 2008, №2, s. 50.

12. Cherevko A.I., Dmitriev B.F., Ryaben'kiy V.M., Muzyka M.M., Soluyanov P.V. Sudovye poluprovodnikovye preobrazovateli. - SPb: SPbGMTU, 2011, $526 \mathrm{~s}$.

13. Zagryadtskiy V.I., Kobylyatskiy N.I., Nedzel'skiy A.P. Ferromagnitnye umnozhiteli chastoty $\mathrm{S}$ vrashchayushchimsya magnitnym polem. Kishinev: «Kartya Moldovenyaske», 1973, $71 \mathrm{~s}$.

14. Kopylov I.P., Gandilyan S.V., Gandilyan V.V. Nekotorye voprosy obobshchennogo fiziko-matematicheskogo modelirovaniya elektromekhanicheskikh preobrazovateley energii - Elektrotekhnika, 1998, №9, s. 25. 